\title{
QUANTITATION OF CALCIUM METABOLISM. THEORY*
}

\author{
BY J.-P. AUBERT, $†$ F. BRONNER, AND L. J. RICHELLE
}

(From the Hospital for Special Surgery and Cornell University Medical College, New York, N. Y.)

(Submitted for publication July 31, 1962; accepted March 1, 1963)

There is general agreement among all who have used $\mathrm{Ca}^{45}$ for kinetic studies in either man or experimental animals that the decrease with time of specific activity of serum calcium can be expressed mathematically by a series of exponential terms. Beyond this, however, opinions diverge, and there is no agreement on either the number of exponential terms needed, the scheme of the body's calcium economy, or the analytical approach for solving the scheme. This paper aims at presenting a general scheme of calcium metabolism, its resolution by a combination of balance and isotope technics, and a critical review of past work.

\section{DEFINITIONS AND NOTATION}

General definitions and notation, those that apply to kinetic analysis generally, regardless of whether and how Ca metabolism is analyzed, are as follows: The pool, $P$, is the sum of two or more compartments, $M$, in units of mass, between which various exchange processes occur. Exchange is the bidirectional movement of ions between two compartments of a pool without detectable change in compartment size. Isoexchange is the movement of ions of the same element, e.g., $\mathrm{Ca}^{45} \rightleftarrows \mathrm{Ca}^{40}$; heteroexchange is that of different elements. This paper will consider only isoionic exchange. Unidirectional entries to and losses from a pool are detectable movements into or out of a pool that may or may not result in a change of pool size. If the amount that enters equals the amount that leaves, pool size does not change, and the pool is in dynamic equilibrium. $V$ designates the rate in units of mass per unit time of an exchange process, and $v$, that of a unidirectional process.

$R$ indicates radioactivity measurements, in counts per minute, or per cent dose; $R_{s}$ indicates specific radioactivity (SA), in counts per minute per milligram $\mathrm{Ca}$, or per cent dose per milligram $\mathrm{Ca}$.

Definitions and notations specific to the scheme of $\mathrm{Ca}$ metabolism shown in Figure 1 follow. The system under analysis is a central $\mathrm{Ca}$ pool, the skeleton and the intestine,

* This work was supported in part by grant A-2209, National Institutes of Health, by grant AT(30-1)-2197, U. S. Atomic Energy Commission, and by the National Dairy Council. Address reprint requests to Dr. F. Bronner, 535 E. 70th St., New York 21, N. Y.

†Guest Scientist: Hospital for Special Surgery and Cornell University Medical College. Permanent address: Institut Pasteur, Paris.

$\ddagger$ Helen Hay Whitney Research Fellow. and is considered to be in steady state, with $v, V$, and $P$ constant dnurig the period of observation. The Ca pool obtains $\mathrm{Ca}$ from the skeleton and food, and loses it to the skeleton and via urine and feces. Various, analyzable exchange processes occur within $P$, which is made up of $M$, $M_{1}, M_{2}$, and $M_{3}$ (Figures 2 and 3 ). The skeleton obtains $\mathrm{Ca}$ from and loses it to $P$. The intestine obtains $\mathrm{Ca}$ from and loses it to the environment and $P$. Balance indicates the difference between input and output, either in the whole system (the body) or in a part (the pool). Traditionally, $\mathrm{Ca}$ balance is that in the system as a whole, or $\mathrm{Ca}$ intake less $\mathrm{Ca}$ output in excreta.

Notation for unidirectional processes and for exchanges, in milligrams per day, is as follows: $\Delta=\mathrm{Ca}$ balance, $\Delta_{P}$ $=\mathrm{Ca}$ balance of pool, $\Delta_{s}=\mathrm{Ca}$ balance of system, $v_{i}=\mathrm{Ca}$ ingested in food, $v_{d}=\mathrm{Ca}$ secreted in digestive juices, $v_{a}=$ $\mathrm{Ca}$ absorbed from intestine, $v_{f}=$ fecal endogenous $\mathrm{Ca}$, $v_{F}=$ total fecal $\mathrm{Ca}, v_{u}=$ urinary $\mathrm{Ca}, v_{o_{+}}=\mathrm{Ca}$ deposited

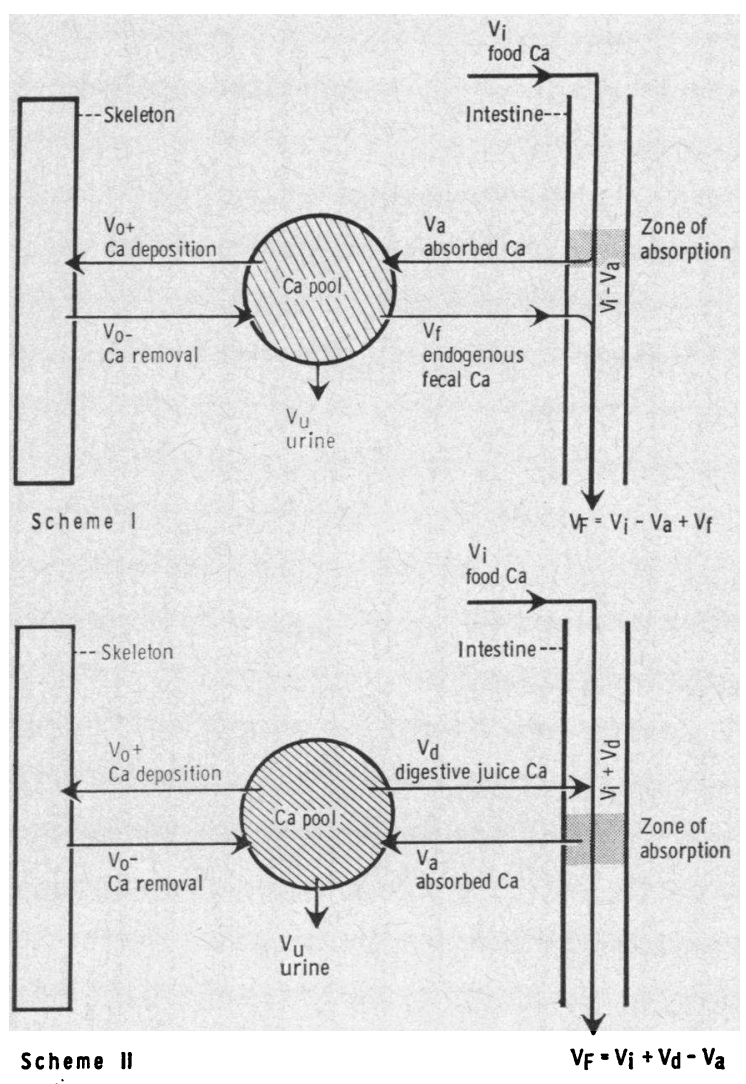

Fig. 1. Two schemes of Ca metabolism. 


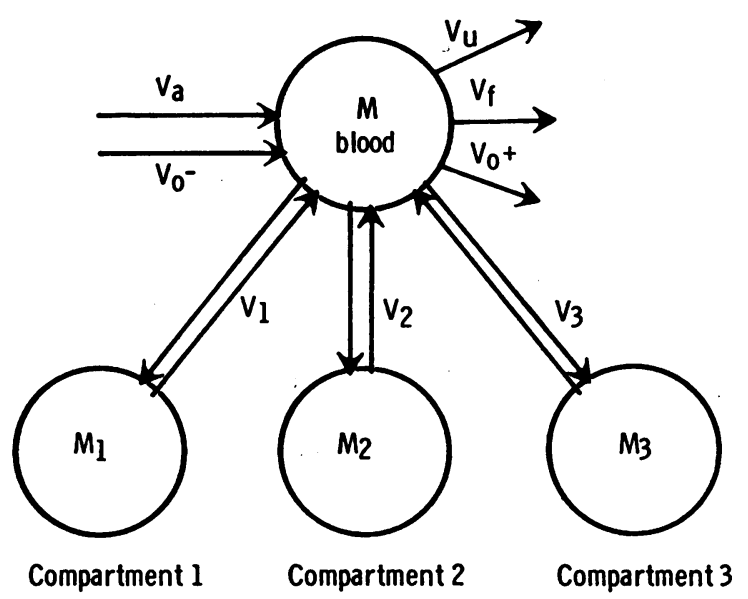

Fig. 2. Model of exchangeable Ca pool. Central compartment, with mass $M$, is identified with blood $\mathrm{Ca}$; identity of remaining three compartments, with masses $M_{1}, M_{2}$, and $M_{3}$, is not specified. Total Ca pool, $P$, equals $M+M_{1}+M_{2}+M_{3}$. All irreversible losses from $P$ are via $M$. Both pool size and rates are considered constant during an experiment. Adapted from Aubert and Milhaud (3).

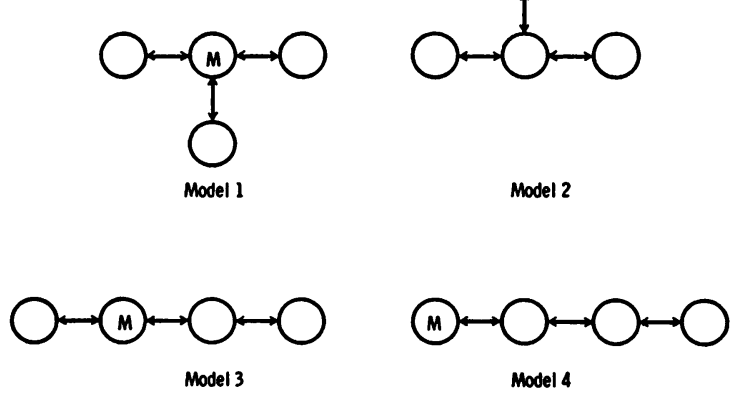

Fig. 3. Different models of Ca exchange.

in skeleton, $v_{o_{-}}=\mathrm{Ca}$ removed from skeleton, and $v_{T}=$ total Ca loss from $P$. Exchanges within $P$ among $M, M_{1}$, $M_{2}$, and $M_{3}$, occur at rates $V_{1}, V_{2}$, and $V_{3}$. Further notation is: $R_{s_{B}}, R_{s_{u}}, R_{s D}$, and $R_{s F}=\mathrm{SA}$ of serum, urinary, digestive-juice, and fecal $\mathrm{Ca}$, respectively, at time $t ; R_{u}$, $R_{D}, R_{F}$, and $R_{o}=$ radioactivity present in urine, digestive juices, feces, and bone, respectively, at time $t ; R_{f}=$ radioactivity due to endogenous fecal $\mathrm{Ca} ; R_{i}=$ radioactivity injected at $t=0 ;$ and $R_{\text {ing }}=$ radioactivity ingested at $t=0$.

\section{DISCUSSION}

\section{Schemes of Ca metabolism}

Figure 1 represents two possible schemes of $\mathrm{Ca}$ metabolism. They are alike in that the system under analysis consists of a central $\mathrm{Ca}$ pool, skeleton, intestine, and loss of $\mathrm{Ca}$ via urine.
They differ in their account of the origin of fecal endogenous Ca. Scheme I pictures fecal endogenous $\mathrm{Ca}$ as secreted into the bowel and not subject to intestinal absorption. This is the simplest way to account for endogenous $\mathrm{Ca}$ in the stool. Scheme II takes more explicit account of what is known of the formation of Ca-containing digestive juices. It derives endogenous fecal $\mathrm{Ca}$ from secretions into the bowel, assuming that the fraction of digestive-juice $\mathrm{Ca}$ absorbed is the same as that absorbed from food. Authors have used either scheme, often without specifying which. Few have studied the entire system; most have studied either absorption or bone metabolism, for example, but not both.

In human studies, Blau and his associates (1) used scheme II and calculated $v_{d}$ and $v_{a}$. Heaney and Whedon (2) used scheme I and calculated $v_{f}, v_{o+}$, and $P$. Aubert and Milhaud (3) and Milhaud, Aubert, and Bourichon (4) also used scheme I, solving for all parameters. Bronner and Harris (5) and also Nordin (6) made no explicit choice of scheme. They calculated $P$, $v_{o+}$, and "endogenous fecal Ca," without further defining the last. Neither Krane, Brownell, Stanbury, and Corrigan (7), nor Bauer, Carlsson, and Lindquist (8), nor Dow and Stanbury (9) indicate a scheme of $\mathrm{Ca}$ metabolism for their calculations of $P$ and $v_{o+}$.

In animal studies, Visek, Monroe, Swanson, Comar, and Hansard $(10,11)$ have calculated $v_{f}$ and $v_{a}$ in the cow, using a scheme like I. Aubert and Milhaud (3) have also used scheme I for their rat studies; as in their human studies, they solved for all parameters. Bauer and coworkers, by defining "exchangeable space" and bone "accretion," have in fact proposed a method for measuring $P, v_{o_{+}}$, and $v_{o_{-}}$in the rat, both in individual bones (12) and in the whole animal (13). For the latter, they had to calculate the loss of $\mathrm{Ca}$ in the excreta, but did not distinguish urinary from fecal endogenous loss.

Of the relationships inherent in the schemes, some are common to both. By definition,

$$
\Delta_{s}=v_{i}-\left(v_{u}+v_{F}\right)
$$

( $\Delta_{s}$ here is identical with traditional Ca balance). If $P$ is in dynamic equilibrium, then

$$
\Sigma v_{\text {out } P}=\Sigma v_{\text {in } P}
$$


in other words, $\Delta_{P}$ must always equal $0 .{ }^{1}$ Since the intestine does not store significant amounts of Ca, it follows, if $\Delta_{P}=0$, that

$$
\Delta_{s}=\Delta_{\text {skel }}=v_{o+}-v_{o-.}
$$

In scheme I, particularly, let $v_{T}$ designate the total Ca loss from $P$ per unit of time. Then,

$$
v_{T}=v_{u}+v_{f}+v_{o+} .
$$

By Equation 2, losses from $P$ must equal gains. Hence,

$$
v_{T}=v_{a}+v_{o-} .
$$

Absorption can also be determined by

$$
v_{a}=v_{i}-v_{F}+v_{f}
$$

and the fraction absorbed is

$$
\alpha=v_{a} / v_{i} \text {. }
$$

In scheme II, some Ca that leaves via $v_{d}$ returns to it via $v_{a}$, but diluted with $v_{i}$. In other words, $v_{F}$ contains some unabsorbed $v_{d}$ and $v_{i}$. The unabsorbed $v_{d}$ is equivalent to $v_{f}$ in scheme I. Hence

$$
v_{f}=v_{d}(1-\alpha) \text {. }
$$

Equation 4 again applies. Equation 5 applies as well, but absorption is also defined by

$$
v_{a}=v_{i}+v_{d}-v_{F},
$$

and the fraction absorbed becomes

$$
\alpha=v_{a} /\left(v_{i}+v_{d}\right) .
$$

Equations 8, 9, and 10 are based on the assumption that $v_{i}$ and $v_{d}$ are absorbed to the same extent and therefore presumably are thoroughly mixed (1).

In terms of actual values, only $v_{a}$ is different in the two schemes. In particular, $\alpha$ is the same in both, since it follows from what was said above that the absorbed fraction of $v_{d}$ is the same as that of $v_{i}$. The principal advantage of scheme II over I is that it allows calculation of what probably is a physiologically meaningful vector, $v_{d}$, whereas $v_{f}$ is physiologically ill-defined.

Although both schemes account nearly equally well for what is known of $\mathrm{Ca}$ metabolism, the

\footnotetext{
1 This does not preclude changes in pool size, but only changes during the period of observation. Changes in pool size between experiments can be observed, e.g., as a result of treatment.
}

conceptualization each represents is meaningful only if based on experimental reality, and the system must be resolvable by available experimental procedures. The system implies the following three conditions.

First, the concept of a central $\mathrm{Ca}$ pool derives from experiments in which $\mathrm{Ca}^{45}$ is injected intravenously, and $R_{s_{B}}$ decreases exponentially with time. In most human studies, $R_{s_{B}}$ decreases monoexponentially between about 36 and 144 hours after iv injection of $\mathrm{Ca}^{45} . \quad R_{s U}$ and $R_{s_{B}}$ are identical during that period $(14,15)$ and the time course of $R_{s_{F}}$ parallels them (15). Since the total serum $\mathrm{Ca}$ concentration is constant, the monoexponential decrease of $R_{s_{B}}$ indicates a system with constant turnover, i.e., a central pool whose $v_{\text {in }}$ and $v_{\text {out }}$ are equal and constant. When the linear portion of the $R_{s_{B}}$ curve is extrapolated to $t=0$, the intercept is much lower than the value obtained by dividing $R_{i}$ by the initial $\mathrm{SA}$ of the blood $\mathrm{Ca}$. Hence, the blood $\mathrm{Ca}$ is in dynamic equilibrium with a much greater $\mathrm{Ca}$ mass, the Ca pool.

Second, there is the constancy of the system during the period of observation. Either the system must be in steady state, or the timevariant parameters must be known. Suitable experimental conditions to keep some of these constant are easily attained and widely practised. Thus, on a metabolic ward, $v_{i}$ can be kept constant, so $v_{u}$ is relatively constant. Although $v_{F}$ will vary from day to day even under these conditions, 6-day collections tend to be fairly constant, particularly if delimited by stool markers. Hence the use of average values on a daily basis for $v_{i}, v_{u}$, and $v_{F}$, based on experimental periods of 6 to 10 days. If $v_{i}$ and $v_{F}$ can reasonably be considered constant under well-defined experimental conditions, it seems as reasonable to suppose that $v_{a}$ and $v_{f}$ can be expressed in terms of mean daily values. If conditions during an experiment are constant, the $\mathrm{Ca}$ content of a subject's blood, extracellular fluid, and other body fluids can be considered constant; it therefore seems unlikely that $P$ varies in that time. This argument is reinforced by the observation of a monoexponential decrease of pool SA during much of the experiment, and by the fact that, in successive experiments on a single patient, variations are generally within the experimental error. If 
$v_{i}, v_{u}, v_{F}, v_{f}, v_{a}$, and $P$ may reasonably be expressed as virtually constant mean daily values, so may $v_{o+}$ and $v_{o-}$.

Finally, since all parameters in a resolvable system can be solved for in their appropriate units of mass, special conditions are imposed on the use of radioactive isotopes. 1) The tracer must be an isotope of $\mathrm{Ca}$, or behave like one. Isotope effects are excluded. 2) In both schemes (Figure 1), it is assumed that during the experimental period either $v_{0-}$ is completely unlabeled, or that a constant fraction of the radioactivity deposited via $v_{o+}$ is returned to $P$. This second possibility has been considered explicitly by Heaney and Whedon (2). Most authors, however, believe that $\mathrm{Ca}$ returned to $P$ by bone resorption is unlabeled. In scheme II, some of the radioactivity lost from the pool via $v_{d}$ is returned to it via $v_{a}$. In order for the SA of $P$ to decrease exponentially under these conditions, it must be assumed that $v_{i}$ and $v_{d}$ undergo rapid mixing and homogenization before absorption, and that the rate of absorption throughout the experiment is relatively constant. 3) Homogenization within each compartment is assumed to be instantaneous. This is not true in fact, but the rate of homogenization is generally very much greater than the rates of exchange between two compartments.

\section{Resolution of system}

The system is resolved by a combination of isotope and balance measurements. In most studies, $\mathrm{Ca}^{r 2}$ has been administered as a single dose either intravenously or by mouth. Although the over-all approach is similar in either case, some assumptions, methods, and the validity of certain of the experimental findings differ. Therefore, it will be useful to discuss both cases separately.

A. Intravenous administration. In human studies, the time-course of $R_{s_{B}}$ is best described by an equation of the type,

$$
\begin{aligned}
R_{s_{B}}=A_{1} e^{-a_{1} t}+A_{2} e^{-a_{2} t} & +A_{3} e^{-a_{3} t}+A_{4} e^{-a_{4} t},
\end{aligned}
$$

which holds for 6 to 7 days after injection of $\mathrm{Ca}^{r}$ $(3,14,16)$. Similar, but three-term equations have been proposed by Krane and associates (7) and Bauer and associates (8). The disappear-

\footnotetext{
${ }^{2}$ Isotope measurements will be designated by $\mathrm{Ca}^{r}$.
}

ance of injected $\mathrm{Sr}$ from the blood has also been described by a four-term equation (17). The first three terms of Equation 11 become negligible within the first 48 hours. Thereafter, the timecourse of $R_{s_{B}}$ is described by

$$
R_{s_{B}}=A_{4} e^{-a_{4} t} .
$$

If $f(t)$ is the time-course of $R_{s_{B}}$ and if all unidirectional losses from $P$ are assumed to be from plasma, then at any time after injected $\mathrm{Ca}^{r}$ has become distributed homogeneously in plasma $\mathrm{Ca}$, a period shown to be less than 1 minute (3), one can write for scheme I :

$$
\begin{gathered}
d R_{u}=v_{u} f(t) d t \\
d R_{F}=v_{f} f(t) d t \\
d R_{o+}=v_{o+} f(t) d t .
\end{gathered}
$$

Upon integration between limits, these equations become,

$$
\begin{aligned}
\left.R_{u}\right]_{t_{1}} t_{2} & =v_{u} \int_{t_{1}}^{t_{2}} f(t) d t \\
\left.R_{F}\right]_{t_{1}} t_{2} & =v_{f} \int_{t_{1}}^{t_{2}} f(t) d t \\
\left.R_{o+}\right]_{t_{1}} t_{2} & =v_{o+} \int_{t_{1}}^{t_{2}} f(t) d t .
\end{aligned}
$$

If follows that

$$
\left.\left.\left.R_{u}\right]_{t_{1}} t_{2} / v_{u}=R_{F}\right]_{t_{1}}{ }^{t_{2}} / v_{f}=R_{o+}\right]_{t_{1}} t_{2} / v_{o+} .
$$

Moreover, at any time $t$,

$$
\begin{aligned}
\left.\left.R_{u}\right]_{o}{ }^{t}+R_{F}\right]_{0} t & \left.+R_{o+}\right]_{o}^{t}+M f(t)+M_{1} g(t) \\
& +M_{2} h(t)+M_{3} i(t)=R_{i}, \quad[16]
\end{aligned}
$$

where $M=$ mass of $\mathrm{Ca}$ in serum, $M_{1}, M_{2}$, and $M_{3}$ are amounts of $\mathrm{Ca}$ in the other compartments of $P$, and $f(t), g(t), h(t)$, and $i(t)$ are the functions with time of SA of $\mathrm{Ca}$ in the various compartments. The same equations apply to Scheme II if $v_{f}$ is replaced by $v_{d}(1-\alpha)$.

Two simplified methods have been proposed on the assumption that $R_{s_{B}}$ is a simple monoexponential function (Equation 12), i.e., that what happens during the first 36 hours after injection can be neglected. The first, that of Bauer and collaborators (8), is based on experiments with rats $(12,13)$, and has been used in human studies by them and other workers $(5,6,8,16,18)$. With it, $v_{f}, v_{o+}$, and $P$ can be determined. The 
method is based on the following relationship, derived from Equation 16:

$$
R_{i}=P f(t)+\left(v_{o+}+v_{u}+v_{f}\right) \int_{o}^{t} f(t) d t
$$

This states that at any given moment, total $R_{i}$ equals the sum of radioactivity remaining in $P$ plus $R_{o}, R_{u}$, and $R_{F} . \quad f(t)$ is set equal to the timecourse of SA. Equation 17 divided by $P$ gives Equation 18:

$$
\begin{aligned}
R_{i} / P= & f(t) \\
& +\left[\left(v_{o+}+v_{u}+v_{f}\right) / P\right] \int_{o}^{t} f(t) d t .
\end{aligned}
$$

If $f(t)$ is a single-term exponential expression and if no labeled $\mathrm{Ca}$ has returned to the pool between times 0 and $t,\left(v_{o+}+v_{u}+v_{f}\right) / P$ equals $a_{4}$ in Equation 12, which is determined experimentally. Equation 18 can then be solved for $P$ :

$$
\left.P=R_{i} /\left[f(t)+a_{4} \int_{0}^{t} f(t) d t\right]\right) .
$$

By measuring the area under the curve described by $\int_{0}^{t} f(t) d t$ and the slope $a_{4}$ on semilog coordinates, $P$ and $a_{4}$ can be calculated; $\left(v_{u}+v_{f}\right)$ is then calculated by applying Equation 14 to the excreta. Hence $v_{o+}$ can be calculated. ${ }^{3}$

In the second simplified method, by extrapolating the linear portion of $R_{s B}$ to $t=0(2,19)$, Equation 12 can be rewritten as:

$$
R_{s_{B}}=\left(R_{i} / P\right) e^{-\left(v_{T} / P\right) t} .
$$

Extrapolating this to $t=0$ gives

$$
R_{8 B}{ }^{o}=R_{i} / P,
$$

hence $P$ can be calculated. Since the slope of Equation $20, v_{T} / P$, is known, $v_{T}$ can be calculated; resolution into its component rates is based on Equations 14a, b, and c.

Two general methods have been proposed that solve a multitermed exponential equation for the entire system. Such methods are important, since substitution of a single-term exponential equation for a multitermed one will give erroneously high values for $P$ and $v$.

In the first, Krane and co-workers (7) chose

\footnotetext{
${ }^{3}$ Bauer and associates (8) have not calculated $v_{f}$ as such, but have calculated $\left(v_{u}+v_{f}\right)$ by Equations 14a and $b$.
}

the following expression to describe the drop of $R_{s_{B}}$ with time:

$$
R_{s_{B}}=A_{1} e^{-k_{1} t}+A_{2} e^{-k_{2} t}+A_{3} e^{-k_{3} t} .
$$

They assume that each exponential term describes the behavior of $\mathrm{Ca}$ in a different compartment, and express $A_{1}, A_{2}$, and $A_{3}$ as the percentage of the dose of $\mathrm{Ca}^{45}$ per gram Ca. They then define the size of each compartment, $Q_{1}$, $Q_{2}$, and $Q_{3}$, as:

$$
\begin{gathered}
Q_{3}=100 \% / A_{3} \\
Q_{2}=100 \% /\left(A_{3}+A_{2}\right) \\
Q_{1}=100 \% /\left(A_{3}+A_{2}+A_{1}\right) .
\end{gathered}
$$

Krane and co-workers (7) do not specify the model underlying their solution, but as they state, Equations 23a, b, and c imply a system where the radioactivity passes from one compartment to the next in successive steps, homogenization in each compartment preceding each step. This hypothesis does not seem likely. Moreover, to approximate the true situation by identifying each term of the equation with a given compartment, rather than Equations 22a, b, and c, one would use the following:

$$
\begin{array}{cc}
Q_{1}=100 \% /\left(A_{1}+A_{2}+A_{3}\right) & {[24 \mathrm{a}]} \\
Q_{1}+Q_{2}=100 \% /\left(A_{2}+A_{3}\right) & {[24 \mathrm{~b}]} \\
Q_{1}+Q_{2}+Q_{3}=100 \% / A_{3} . & {[24 \mathrm{c}]}
\end{array}
$$

Equation $24 \mathrm{c}$ is merely another way of calculating $P$ by the simplified method, e.g., with Equation 20.

In the second general method, Aubert and Milhaud (3) express the time-course of $R_{s_{B}}$ by Equation 11. The analysis assumes that the blood represents the central compartment whose $\mathrm{Ca}$ exchanges simultaneously with that of the other compartments [see also (20)]. Then, $A_{1}$, $A_{2}$, etc., and $a_{1}, a_{2}$, etc., represent functions of both each $V$ and each $M$. These functions are complex, and a given term of the equation cannot be identified with a particular compartment. Aubert and Milhaud (3) have shown experimentally that the blood constitutes a well-defined compartment for $\mathrm{Ca}$ exchange. They measured the time-course of $R_{s_{B}}$ during the first 90 seconds after injection and showed that a maximum occurs at about 45 seconds. This maximum 
amounted to $75 \%$ of the theoretical maximal value, $R_{i} / M_{\text {blood. }}$ Moreover, if the nearly linear decrease of the $R_{s_{B}}$ curve between 45 and 90 seconds after injection was extended to $t=0$, it intercepted the ordinate at a point within $5 \%$ of the calculated values for $R_{i} / M_{\mathrm{blood}}$, as in their Figure 5 (3).

This type of analysis requires a well-defined model of compartments and rates, and the model shown in Figure 2 was chosen; it represents a mammillary system with the blood as the central compartment. The system is resolved as follows. Let $R_{i}=$ the amount of $\mathrm{Ca}^{r}$ injected at $t=0$, and $R, R_{1}, R_{2}$, and $R_{3}=$ the amount of $\mathrm{Ca}^{r}$ in the blood, and in compartments 1,2 , and 3 , respectively, at time $t$. Also, let $d R, d R_{1}, d R_{2}$, and $d R_{3}$ represent the change in the amount of $\mathrm{Ca}^{r}$ per time $d t$. The dynamic state of the system can then be expressed by the following equations:

$$
\begin{gathered}
d R / d t=-\left[\left(V_{1}+V_{2}+V_{3}+v_{T}\right) / M\right] R \\
+\left(V_{1} / M_{1}\right) R_{1}+\left(V_{2} / M_{2}\right) R_{2}+\left(V_{3} / M_{3}\right) R_{3} \\
d R_{1} / d t=\left(V_{1} / M\right) R-\left(V_{1} / M_{1}\right) R_{1} \\
d R_{2} / d t=\left(V_{2} / M\right) R-\left(V_{2} / M_{2}\right) R_{2} \\
d R_{3} / d t=\left(V_{3} / M\right) R-\left(V_{3} / M_{3}\right) R_{3} .
\end{gathered}
$$

Knowing $M$, the total blood Ca content, one can then calculate all other unknowns and thereby resolve the system.

Besides the assumptions discussed above (see Discussion, I), this analysis also assumes that all unidirectional losses from $P\left(v_{u}, v_{F}\right.$, and $\left.v_{o+}\right)$ occur by way of the central compartment. It seems reasonable to equate $v_{o_{+}}$, the unidirectional loss of $\mathrm{Ca}$ from $P$ into bone, with bone formation. As long, however, as a formal distinction is maintained between bidirectional $\mathrm{Ca}$ exchange and unidirectional $\mathrm{Ca}$ movement from pool to bonei.e., bone formation and its component processes - the analysis described here and by schemes I and II (Figure 1) is independent of the nature of this unidirectional process (see also Discussion, III).

This type of analysis raises an important point, the kind of model into which the pool is decomposed. Equation 11 represents a function by which the plasma $\mathrm{Ca}$ appears to exchange at three different rates with three different $\mathrm{Ca}$ compartments of varying size. The equation does not, however, indicate how the compartments of $P$ are related. Four models are possible (Figure 3), each resolved differently and leading to different results. One or more additional exchanges may occur, so that the model includes one or several cycles. Under those conditions, the model can no longer be solved. Unidirectional entries and losses may also occur via a compartment other than the central one. Although resolution of such models is feasible, it becomes more complex.

Aubert and Milhaud (3) have advanced arguments in favor of model 1 (Figure 3), but model 2 seems equally reasonable from what is known of $\mathrm{Ca}$ metabolism, particularly as in normal patients both models give similar values. Once, however, all unidirectional movements are assumed to occur by way of the blood compartment ( $M$ in Figure 3 ), the model chosen affects only the manner in which the $\mathrm{Ca}$ of $P\left(M+M_{1}\right.$ $\left.+M_{2}+M_{3}\right)$ is partitioned among the three other compartments (with the blood $\mathrm{Ca}$ constant), but has almost no effect on the values calculated for $v_{T}$ and its component rates. When experimental results from a normal person, a patient with Paget's disease, and one with hypothyroidism were calculated according to scheme I (Figure 1), it was shown (3) that rates and compartment sizes varied considerably, but that the rate constants (rate/mass) varied less, indicating that the same exchange process was measured in all three cases. Aubert and Milhaud (3) postulate that $M_{1}$ is the $\mathrm{Ca}$ of the extracellular fluid, $M_{2}$ is the more labile portion of the tissue $\mathrm{Ca}$, and $M_{3}$ is the less labile tissue $\mathrm{Ca}$ plus the exchangeable $\mathrm{Ca}$ of bone.

The relative error that arises in the simplified as compared to the general method can be estimated by neglecting the first three terms of Equation 11 [see (3) for theoretical discussion]. In all instances, values of $P$ and $v_{o+}$ calculated by the simplified method are larger than the true values obtained by the general method, with the error becoming relatively larger when $P$ and $v_{o+}$ are large. Hence, use of the simplified method is justified only when $P$ is small, or in comparative studies.

Animal studies. Resolution of the disappearance equations obtained in animal experiments can be treated as in human studies. In the first of two methods of resolution, both developed in 
work with rats, Bauer and associates $(12,13,22)$ have shown that in a clearly defined portion of the diaphysis of the tibia, the $\mathrm{Ca}^{45}$ content, $\left.R_{o}\right]_{0}{ }^{t}$, is given by

$$
\left.R_{o}\right]_{o}{ }^{t}=k_{1} f(t)+k_{2} \int_{o}^{t} f(t) d t
$$

where $f(t)$ is a function of $R_{s_{B}}, k_{1}=$ the exchangeable $\mathrm{Ca}$ of the bone, and $k_{2}=$ the rate of bone formation. Bauer and co-workers calculated $k_{1}$ and $k_{2}$ of Equation 26 by taking as reference the numerical value of Equation 264 hours after the rats were injected, and compared values for $k_{1}$ and $k_{2}$ obtained subsequently $(8,16,24$, and up to 120 hours). They thus obtained a series of linear equations with two unknowns and a series of values for $k_{1}$ and $k_{2}$. These were fairly constant for the bone shafts up to 120 hours, but were constant for the bone ends only during the first 48 hours [see their Table III (22)]. Resorption was measured by weighing bone and determining its $\mathrm{Ca}$ content at various times. Resorption, $v_{o_{-}}$, must then equal the difference between $v_{o+}$ as calculated above and the bone balance. Excretion from the body was measured by determining the total $R_{u}$ and $R_{F}$ and applying Equations 14a and b.

This method is theoretically sound, and is independent of the type of function that $R_{B_{B}}$ may have, since the $\mathrm{Ca}^{45}$ content of the bone is measured directly. The method cannot, however, be extended to measure all the parameters of scheme I or II in accordance with Equation 18, since in rats the time-course of $R_{s_{B}}$ cannot be correctly represented by a monoexponential function $(3,20)$.

Other authors $(3,23,24)$ have applied the general method of resolution described for studies with humans to studies with rats. Hence all parameters of either scheme I or II can be calculated without killing a large number of animals. According to Milhaud, Remagen, Gomes de Matos, and Aubert (23), $R_{s_{B}}$ in rats between 0 and 72 hours after injection can be described adequately by a two-term exponential equation of the type,

$$
R_{8 B}=A_{1} e^{-a_{1} t}+A_{2} e^{-a_{2} t},
$$

which is then solved with the aid of a two-compartment model. Aubert and Milhaud (3) have shown experimentally that the calculated values for $v_{o+}$ agree well with experimental values obtained by analysis of the whole skeleton. Bronner (20) used a model of $\mathrm{Ca}$ metabolism like that shown in Figure 2. He compared $\mathrm{Ca}$ uptake of the skeleton with that in certain tissues and organs. By calculating constants and fitting them to equations similar to Equations 25a, b, and c, he was able to show that the model adequately represented the experimental data. He did not, however, resolve the constants into absolute rates and compartment sizes.

B. Oral administration. Several authors have attempted to measure $\mathrm{Ca}$ absorption in man (1, $5,15,25)$ or animals $(10,11)$ by giving $\mathrm{Ca}^{r}$ orally. At first glance, this seems the simplest and most direct approach to measuring absorption, but it involves major difficulties of interpretation and technique. If $\alpha=$ fraction of $\mathrm{Ca}$ that is absorbed, then the radioactivity absorbed, $R_{a}$, is given by

$$
R_{a}=\alpha R_{\text {ing }}
$$

Obviously, the metabolic processes affecting $\mathrm{Ca}$ are identical for exogenous and endogenous Ca. Therefore, the physiological behavior of absorbed $\mathrm{Ca}$ can be analyzed as though $\mathrm{Ca}$ were injected intravenously. Then, if $\alpha$ can be determined, the system can be solved.

In one method of determining absorption, no intravenous experiment precedes the oral experiment. Analysis of this case according to scheme I follows. Equation 6 applied to radioactivity measurements ${ }^{4}$ gives

$$
\left.\left.R_{a}=R_{\text {ing }}-R_{F}\right]_{o}^{t}+R_{f}\right]_{o}^{t} .
$$

Equations 14a and $\mathrm{b}$ combined give

$$
\left.\left.R_{f}\right]_{o}^{t}=\left(v_{f} / v_{u}\right) R_{u}\right]_{o}^{t},
$$

which, when combined with Equation 29, gives

$$
\left.\left.R_{a}=R_{\text {ing }}-R_{F}\right]_{o}^{t}+\left(v_{f} / v_{u}\right) R_{u}\right]_{o}{ }^{t} . \quad[31]
$$

Since it can be assumed that the same fraction of $\mathrm{Ca}^{r}$ and $\mathrm{Ca}$ is absorbed,

$$
\begin{array}{r}
\alpha=\left(v_{i}-v_{F}+v_{f}\right) / v_{i}=\left(R_{\text {ing }}-R_{F}\right]_{o}^{t} \\
\left.\left.+\left(v_{f} / v_{u}\right) R_{u}\right]_{o}^{t}\right) / R_{\text {ing. }} .
\end{array}
$$

4 When $\mathrm{Ca}^{r}$ is given intravenously, $R_{f} \equiv R_{F}$; when $\mathrm{Ca}^{r}$ is given orally, these two values must be differentiated. 
From this, $v_{f}$ and $\alpha$ can be calculated as follows:

$\left.v_{f}=v_{u}\left(v_{F} R_{\text {ing }}-v_{i} R_{F}\right]_{o}{ }^{t}\right) /$

$$
\left.\left(v_{u} R_{\text {ing }}-v_{i} R_{u}\right]_{o}\right)^{\prime}
$$

$\left.\alpha=1-\left[\left(v_{u} R_{F}\right]_{o}{ }^{t}-v_{F} R_{u}\right]_{o}{ }^{t}\right) /$

$$
\left.\left.\left(v_{u} R_{\text {ing }}-v_{i} R_{u}\right]_{o}{ }^{t}\right)\right] \text {. }
$$

In the analysis according to scheme II, Equations 9 and 10 are combined and applied to the radioactivity measurements, as in Equation 29. The value for $\left.R_{D}\right]_{o}{ }^{t}$ is substituted, by analogy with Equation 30, to give:

$$
\begin{array}{r}
\left.R_{F}\right]_{0}{ }^{t}=(1-\alpha) R_{\text {ing }} \\
\left.+(1-\alpha) R_{u}\right]_{o}{ }^{t}\left(v_{d} / v_{u}\right) .
\end{array}
$$

This can also be derived from Equation 31 by substituting $v_{d}(1-\alpha)$ for $v_{f}$. Since it is independent of the scheme used, Equation 34 defines $\alpha$. Equation 36, derived from Equations 34 and 35 , gives $v_{d}$ :

$$
\begin{array}{r}
\left.v_{d}=v_{u}\left(v_{i} R_{F}\right]_{o}^{t}-v_{F} R_{\mathrm{ing}}\right) / \\
\left.\left.\left(v_{F} R_{u}\right]_{o} t-v_{u} R_{F}\right]_{o}^{t}\right) .
\end{array}
$$

If $R_{F}$ of $\mathrm{Ca}^{r}$ is recorded for more than 5 to 7 days, unabsorbed $\mathrm{Ca}^{r}$ will have been excreted in the stool ; $\mathrm{Ca}^{r}$ then excreted in the stool is endogenous, and analysis of this latter part of the curve can proceed as for iv injection.

In a second method for determining absorption, the oral experiment can be compared with an intravenous one. If an iv experiment is performed shortly before or after the absorption study, data of both studies can be combined to calculate $\alpha$, on the assumption that no significant metabolic change has occurred between the two studies. Two methods of calculating absorption in this way have been proposed. Both are based on Equation 28 and are independent of the scheme (I or II) used. First, the urine method [method B in (26)] is based on the fact that in a stable system, $R_{u}$ of $\mathrm{Ca}^{r}$ is proportional to the amount introduced into the system, or:

$$
\left.\left.\alpha=\left[\left(R_{u}\right]_{o} t / v_{u}\right)_{\text {ingested }} /\left(R_{u}\right]_{o} t / v_{u}\right)_{\text {injected }}\right],
$$

where $t \cong 6$ days. Second, in the pool method (4), $P$ is calculated by the simplified method, by extrapolating the linear portion of the disappearance curve to the origin as if absorption were complete:

$$
\alpha=P_{\text {injection }} / P_{\text {ingestion }} .
$$

In either method, $\alpha$ can be calculated without the need for a $\mathrm{Ca}$ balance, in either the intravenous or the oral part of the experiment. The two methods are not fully equivalent, however, since they rest on somewhat different assumptions. Let $\alpha_{r}$ be the real and $\alpha_{m}$ the measured value of $\alpha$; and let $P$ and $v_{T}$ be values in an injection experiment, and $P^{\prime}$ and $v_{T}{ }^{\prime}$ be values in an oral experiment. If $R_{B_{B}}$ is considered a monoexponential function, Equations 37 and 38 then become, respectively, in the urine and pool methods:

$$
\begin{gathered}
\alpha_{m}=\alpha_{r}\left(v_{T} / v_{T}{ }^{\prime}\right)\left[1-e^{-\left(v_{r^{\prime}} / P^{\prime}\right) t} /\right. \\
\left.1-e^{-\left(v_{r} / P\right) t}\right] \\
\alpha_{m}=\alpha_{r}\left(P / P^{\prime}\right) .
\end{gathered}
$$

In the urine method, $\alpha_{m}=\alpha_{r}$ if $P=P^{\prime}$ and $v_{T}=v_{T}{ }^{\prime}$. The value of $e^{-\left(v_{T} / P\right) t}$ in Equation 39 is small, however, when $t=6$ days. Hence the relationship between $\alpha_{m}$ and $\alpha_{r}$ is determined essentially by $v_{T} / v_{T}{ }^{\prime}$. In the pool method, on the other hand, the relationship between $\alpha_{m}$ and $\alpha_{r}$ is determined only by $P / P^{\prime}$ and is completely independent of the rates of turnover of the pool. Nevertheless, if $v_{T} / P$ remains constant in the two experiments, as measurement of $R_{s_{w}}$ can easily verify, the urine method is better than the pool method, since it is independent of the type of function (monoexponential or not) that may represent the time-course of $R_{s_{u}}$. Moreover, when $P$ is determined by the simplified method, the error can be fairly large (see above).

General resolution of the system is possible and all parameters (by either scheme I or II) can theoretically be solved for in an oral experiment. If a tracer dose of $\mathrm{Ca}^{r}$ is added to a test meal, general resolution of the system is possible by the radioactivity balance method, even if comparison with an iv experiment is not feasible. It is necessary to subject the patient to the usual balance study to determine $v_{u}, v_{F}, v_{i}$, and $\Delta$. In scheme I, $v_{f}$ and $\alpha$ are calculated from Equations 33 and 34. By using the simplified method and extrapolating the curve of the logarithm of $R_{s_{w}}$ to $t=0$, one can calculate a $P_{\max }$ and $v_{T_{\max }}$ as if absorption were complete. The true $P$ and $v_{T}$ can then be calculated by setting

and

$$
P=\alpha P_{\max }
$$

$$
v_{T}=\alpha v_{T \max } .
$$


Since $v_{u}$ is determined experimentally and $v_{f}$ is calculated by Equation 33, one can solve for $v_{o+}$ by Equation 4 and for $v_{o_{-}}$by Equation 3 once $\Delta$ is determined. Finally, $v_{a}$ is given by Equation 7. In scheme II, $\alpha$ and $v_{d}$ are given by Equations 34 and 36, respectively, and $P$ and $v_{T}$ are then calculated as above, by Equations 41 and 42. Values of $v_{f}, v_{o+}, v_{o_{-}}$, and $v_{a}$ are given by Equations 8, 4, 3, and 9, respectively.

When comparison with an iv experiment is possible, combination of either the pool or the urine method with a $\mathrm{Ca}$ balance study gives $\alpha$, $P, v_{T}, v_{u}, v_{i}$, and $\Delta$. In scheme I, $v_{a}, v_{o_{-}}, v_{o+}$, and $v_{f}$ are given by Equations 7, 5, 3, and 4, respectively. In scheme II, combination of Equations 9 and 10 gives $v_{d}$ and $v_{a} ; v_{f}, v_{o+}$, and $v_{o-}$ are given by Equations 8, 4, and 3, respectively.

In the case of $\mathrm{Ca}$ absorption of a $\mathrm{Ca}^{r}$-labeled compound, a $\mathrm{Ca}$ balance has no meaning unless all dietary $\mathrm{Ca}$ is in the form of the labeled compound. This can be done in animal, but not easily in human studies. For resolution of the system, data from an iv study must be used. Since, however, $\alpha$ calculated for the labeled compound need bear no relation to $\alpha$ of the food in general, resolution of the system may not be meaningful.

On the other hand, if absorption of a specific $\mathrm{Ca}$ compound is to be compared with that of dietary $\mathrm{Ca}$ in general, experiments (by both injection and ingestion can be performed simultaneously with two different isotopes of $\mathrm{Ca}$, e.g., $\mathrm{Ca}^{45}$ and $\mathrm{Ca}^{47}$ (26). The $\alpha$ of the $\mathrm{Ca}$ compound can then be calculated by either urine or pool method.

Oral studies have both theoretical and physiological limitations. To resolve all parameters of either scheme I or II after oral administration of $\mathrm{Ca}^{r}$, one must assume that $\alpha R_{\text {ing }}$ behaves like an equivalent amount of $R_{i}$. Absorption is not an instantaneous process; rather, it takes 2 to 3 hours before $R_{s_{B}}$ reaches its peak after an oral dose (27). The error becomes even larger with the simplified method, which presupposes instantaneous homogenization of the entire pool. Sufficiently frequent blood sampling after ingestion of tracer, however, could establish an equation of the type:

$$
\begin{gathered}
R_{s_{B}}=-\left(A_{1}+A_{2}+A_{3}+A_{4}\right) e^{-a t}+A_{1} e^{-a_{1} t} \\
+A_{2} e^{-a_{2} t}+A_{3} e^{-a_{3} t}+A_{4} e^{-a_{4} t} \cdot
\end{gathered}
$$

From this, the system could be resolved, since Equation 43 takes into account the time of absorption.

There are also physiological considerations. To use an oral study for the resolution of the system, one must assume all dietary $\mathrm{Ca}$ to be labeled homogeneously before the study. Since this is impractical, $\mathrm{Ca}^{r}$ is usually added to milk (27) or to a particular $\mathrm{Ca}$ compound, like a $\mathrm{Ca}$ salt (28). Absorption of milk Ca must be assumed not to differ from that of the general dietary $\mathrm{Ca}$, an assumption that remains unproved. In the second case, one can measure $\mathrm{Ca}$ absorption of the salt in question, but cannot, as shown above, resolve the entire system. Oral experiments, therefore, do not appear to be suitable for study of $\mathrm{Ca}$ metabolism in general. The absorbability of $\mathrm{Ca}$ in specific foods or compounds can be readily determined in conjunction with an iv study.

\section{Long-term behavior of $R_{s_{B}}$.}

All the human studies discussed so far involve periods of 6 to 8 days after isotope administration. Then the monoexponential decrease of $R_{s_{B}}$ observed between 2 and 6 to 7 days ceases, and the curve assumes a new slope. Two interpretations of this phenomenon have been offered.

Heaney and Whedon (2) believe the new and flatter slope of the $R_{s_{B}}$ curve, seen about 1 week after injection of radioactivity, is due to the return of label into $P$, resulting from resorption of labeled bone after a period $\theta$. Dansky and Heaney [quoted in (2)] have derived a mathematical expression that accounts for recycling in an open single-compartment system:

$$
x_{n}(t)=\sum_{n=0}^{N} \frac{x_{0}(0) e^{K(t-n \theta)}\left[k_{b}{ }^{\prime}(t-n \theta)\right]^{n}}{n !},
$$

where $x_{0}=$ the specific radioactivity at $t=0$, $K=$ the rate constant of loss from the pool, $k_{b}{ }^{\prime}$ $=$ the rate constant of loss from bone, and $\theta=$ time interval required for recycling the tracer. Heaney and Whedon (2) have applied Equation 44 at the end of the second cycle-i.e., for $2 \theta$ and when $n=1$-in order to calculate the fraction of $v_{o+}$ that returns to $P$ under these conditions. This fraction was found to vary between 20 and $40 \%$, with a mean of $32 \%$. 
Aubert and Milhaud (3) attribute the same break observed in the $R_{s_{B}}$ curve to a slow, physicochemical phenomenon. They propose to solve for it by adding a fifth compartment, $M_{4}$, to the four-compartment mammillary exchange system shown in Figure 2. According to their calculations, the true $v_{o_{+}}$is about $25 \%$ smaller than that calculated by the general methods discussed above.

Although both Heaney and Whedon, and Aubert and Milhaud arrive at similar estimates of the magnitude of this slow phenomenon, they differ profoundly in interpretation. Heaney and Whedon consider it to be due to bone resorption; Aubert and Milhaud [also Krane and associates (7)] attribute it to a slow exchange. Only further studies can resolve this point, especially in the light of the "slow exchange process" described by Marshall, Rowland, and Jowsey (29) in vivo and by Neuman and Neuman (30) in vitro. The process described by Marshall and associates, however, is more akin to a translocation of $\mathrm{Ca}$ in the skeleton without measurable effect on $P$ and is in no way comparable to exchange as defined here.

\section{Special methods of system analysis}

In $\mathrm{Ca}^{r}$ infusion studies, Rich, Ensinck, and Fellows $(31,32)$ have attempted to correlate estimated changes in pool size with clinical states. Rich (31) administered a mixture of $\mathrm{Ca}$ gluconate and $\mathrm{Ca}$ glycerophosphate labeled with $\mathrm{Ca}^{r}$ at a constant rate (110 $\mathrm{mg}$ Ca per hour) for 4 hours, and measured radioactivity in serum and excreta. $P$ and $v_{o+}$ were calculated by the method of Bauer and associates (8). The size of the rapidly exchanging $\mathrm{Ca} P$ was calculated by use of the curve representing increase in $R_{s B}$ during infusion. Rich defined this $P$ at time $t$ by the relationship: $E_{R}=$ total radioactivity injected by time $t /$ serum $S A$ at time $t$ (Equation 45). In later studies (32), high-specific-activity $\mathrm{Ca}^{45}$ in isotonic saline was infused by a constant-rate pump, and the "miscible calcium" was defined as the rate at which $\mathrm{Ca}^{45}$ was administered divided by the slope of the regression curve fitted to the rising specific-activity values measured during the experiment. In these studies, only "miscible Ca" was determined.
To use the method of Bauer and associates (8) for calculating $P$ and $v_{o_{+}}$, it is essential to define a zero time. This cannot be done precisely in Rich's method, and his later studies (32) abandon this approach. $E_{R}$, as defined by Equation 45 , is not only a function of the size of exchangeable $P$, but equally a function of all the rates. Whatever scheme is chosen to resolve the system, if two subjects have the same size pool, but different rates of exchange, the serum $\mathrm{Ca}$ will undergo exchange with different quantities of $\mathrm{Ca}$ during the infusion. Consequently, $E_{R}$ will differ in the two cases, even though the pools are in fact identical. This argument applies also to the rates of irreversible loss and gain of the central compartment, but over a 4-hour period, these are small in comparison to the rates of exchange.

There is also no way of predicting at present the effect of the $\mathrm{Ca}$ infusion $(110 \mathrm{mg} \mathrm{Ca}$ per hour) on the parameters of the system, even if serum $\mathrm{Ca}$ concentration is unchanged. In the later studies, however, the total $\mathrm{Ca}$ infused was negligible $(\cong<30 \mu \mathrm{g} \mathrm{Ca})$.

Despite these criticisms of the theoretical significance of the $E_{R}$, it is interesting to note that the rate constants $V_{1} / M_{1}, V_{2} / M_{2}, V_{3} / M_{3}$ are relatively invariant (3). Hence, a major change in $M$ is likely to be accompanied by a comparable change in $V$. This may explain why Rich was able to distinguish by this test major alterations in $E_{R}$ associated with certain diseases.

In another analysis, Krane and associates (7) define the Ca pool of man at time $t$ as: $P_{t}=$ body retention of radioactivity at time $t /$ serum $S A$ at time $t$ (Equation 46). As defined, this "pool" must necessarily be a function of time. Krane and associates, however, claim that $P_{t}$ approaches a limit at $t=9$ days, thus permitting a comparison of $P_{t(9 \text { days })}$ in different patients. They state: "The concept of a calcium 'pool' makes no assumption based on the shape of the curves, but reflects the fact that a lower specific activity at any time means a larger mass of dilution." (7).

To examine this statement more closely, let us consider that $R_{s_{B}}$ decreases monoexponentially. Equation 46 then becomes:

$$
\begin{aligned}
\left.P_{t}=\left[R_{o}\right]_{o} t+P\left(R_{s_{B}}\right)_{t}\right] /\left(R_{s_{B}}\right)_{t} & \left.=P+R_{o}\right]_{o} t\left(R_{s_{B}}\right)_{t} .
\end{aligned}
$$


Using Equations $14 \mathrm{c}$ and 20, one can write

$$
\begin{gathered}
\left.R_{o}\right]_{o} t /\left(R_{s_{B}}\right) t=\left[R_{i}\left(v_{o+} / v_{T}\right)\left(1-e^{-\left(v_{T} / P\right) t}\right)\right] / \\
\quad\left(R_{i} / P\right) e^{-\left(v_{T} / P\right) t}=P\left(v_{o+} / v_{T}\right)\left(e^{\left(v_{T} / P\right) t}-1\right),
\end{gathered}
$$

so that Equation 47 becomes

$$
P_{t}=P\left[1+\left(v_{o+} / v_{T}\right)\left(e^{\left(v_{T} / P\right) t}-1\right)\right] .
$$

In other words, the "pool at time $t$ " becomes a rather complex function of $P$ and of $v_{o+}$ and $v_{T}$. Moreover, $P_{t}$ increases indefinitely with time. The notion of a "pool at time $t$ " does not add to what can be learned by solving the system.

The excretion index defined by Bronner and associates $(33,34)$ consists of three parameters after iv injection of $\mathrm{Ca}^{r}$ where $t=$ days:

$$
\begin{aligned}
A & \left.=R_{u}\right]_{o}{ }^{6 t} \\
B & \left.=v_{u}\right]_{o}{ }^{6 t} \\
C & =A / B .
\end{aligned}
$$

Rewritten in terms of Equation 14a:

$$
\begin{aligned}
& A=v_{u} \int_{0}^{6} f(t) d t \cong R_{i}\left(v_{u} / v_{T}\right) \\
& B=6 v_{u} \\
& C=(1 / 6) \int_{0}^{6} f(t) d t \cong\left(R_{i} / v_{T}\right) .
\end{aligned}
$$

The integral in Equation 51 is independent of the nature (mono- or multiexponential) of the SA curve. Values of $A, B, C$ can be obtained simply by measuring $S A$, Ca concentration, and the volume of 6 successive 24-hour urine samples after iv injection of tracer. The way these parameters vary indicates variations of $v_{u}$ and $v_{T}$. Since $v_{o+}$ is the major component of $v_{T}$, variation in the excretion index suggests how $v_{u}$ and also $v_{o+}$ are affected. Although the excretion index does not give a complete description of Ca metabolism, it can be useful in clinical screening studies, e.g., to evaluate the effect of drugs.

In a recent preliminary publication, Bhandarkar, Bluhm, MacGregor, and Nordin (35) propose to measure $\mathrm{Ca}$ absorption by giving patients labeled $\mathrm{CaCl}_{2}$ and measuring $R_{s_{B}}$ after 2 hours. To support the claim that $R_{s_{B}}$ is a measure of absorption, these workers plotted $R_{s_{B}}$ against "net calcium absorption," $\left(v_{i}-v_{F}\right)$, and observed a linear relation with an error of approximately $\pm 42 \%$. Clearly, such a test provides only an indication, not a true measure, of absorption; it may be useful in certain screening studies.

\section{CONCLUSION}

The preceding discussion has shown that a number of conditions must be met to obtain meaningful answers in a kinetic analysis of $\mathrm{Ca}$ metabolism. a) There are experimental conditions for isotope administration. To study a system in a steady state, the tracer introduced must perturb it as little as possible. Since the blood represents a well-defined compartment, highspecific-activity $\mathrm{Ca}^{r}$ is best administered by rapid, iv injection. In other words, the mass of $\mathrm{Ca}^{r}$ introduced must be negligibly small in relation to that of the blood.

b) The disappearance curve must be established. The precision of measurement of this fundamental parameter is a function of the number of measurements made, particularly during the early part of the curve, when it falls off rapidly. When the general method of resolution is used in human studies, blood samples should be obtained at $2,4,8,15,30,45,60,75,120,180$, 360,540 , and 720 minutes, and 24 and 48 hours after injection. The rest of the curve is more easily obtained by measuring $R_{s_{u}}$. The size of the blood $\mathrm{Ca}$ compartment is determined from plasma volume. The simplified method may be used when the patient is a small child, or when many patients are being studied for comparative purposes and their pools are known to be small. Then, all determinations may be of $R_{s_{u}}$.

c) To measure $\mathrm{Ca}$ intake and output, the diet must be weighed and its $\mathrm{Ca}$ content determined. Measurement of urinary $\mathrm{Ca}$ and $\mathrm{Ca}^{r}$ output presents no difficulties. The principal problem with fecal $\mathrm{Ca}$ output is to establish the correct time correspondence between intake and output, in terms of both balance and kinetic measurements. Since $P$ becomes homogenized between 36 and 48 hours after injection, the simplest method is to give a suitable marker, e.g., $1 \mathrm{~g}$ carmine, at 36 hours, e.g., at the evening meal of day 2, and to collect all stools separately thereafter. Six days later, another marker is given. Total $\mathrm{Ca}$ and $\mathrm{Ca}^{r}$ is then measured in all stools for that period, i.e., from the stool with 
the first trace of the first marker through, but excluding, the stool with the first trace of the second marker.

For analysis of results, the general method of resolution best minimizes all sources of error (see Discussion, II). Choice of model (Figure 3) will affect primarily the determination of $M$ and $V$ within $P$, and only slightly that of unidirectional processes. Consequently, an investigator may treat data according to more than one model; if this is done systematically, it may indicate an ultimate choice of model. At present, experimental evidence does not permit a clearcut choice between schemes I and II (Figure 1), but as data accumulate, one scheme or the other will be preferred for consistent interpretation. Again, data may be treated according to both schemes.

Although the simplified method leads to erroneously high values for $P$ and $v_{o+}$, it can be used in comparative series where the true $P$ is small, and where greater simplicity of analysis and calculation becomes important. The simplified method also has a place as a diagnostic tool in a metabolic ward, provided its limitations are taken into account.

In absorption studies, oral administration of $\mathrm{Ca}^{r}$ is theoretically valid only to measure $\mathrm{Ca}$ absorption for a given $\mathrm{Ca}$ compound, or a single, (uniformly labeled) Ca-containing food. For this, data from an iv study must be available. Under these conditions, $\alpha$ is best calculated by the urine method (Equation 37). In screening studies on the effect of a drug on Ca metabolism, evaluation of the excretion index is the most economical method (see Discussion, IV).

\section{SUMMARY}

A detailed, formal analysis of $\mathrm{Ca}$ metabolism has been presented that permits measurement of sizes and exchange rates of compartments constituting the $\mathrm{Ca}$ pool, as well as the following unidirectional rates: bone formation and resorption, absorption, urinary $\mathrm{Ca}$ output, and endogenous fecal $\mathrm{Ca}$ loss. The last can also be expressed as a fraction of the rate of formation of digestive juice $\mathrm{Ca}$.

Two possible schemes of $\mathrm{Ca}$ metabolism have been presented and analyzed; these differ in their account of the origin of endogenous fecal $\mathrm{Ca}$.
Calculations according to either scheme are detailed.

Both general and special methods of analysis of $\mathrm{Ca}$ metabolism have been presented, their advantages and drawbacks discussed, and their applicability evaluated.

\section{ACKNOWLEDGMENT}

The encouragement and support of Dr. Philip D. Wilson, Hospital for Special Surgery, are gratefully recognized.

\section{REFERENCES}

1. Blau, M., H. Spencer, J. Swernov, J. Greenberg, and D. Laszlo. Effect of intake level on the utilization and intestinal excretion of calcium in man. $\mathrm{J}$. Nutr. 1957, 61, 507.

2. Heaney, R. P., and G. D. Whedon. Radiocalcium studies of bone formation rate in human metabolic bone disease. J. clin. Endocr. 1958, 18, 1246.

3. Aubert, J.-P., and G. Milhaud. Méthode de mesure des principales voies du métabolisme calcique chez l'homme. Biochim. biophys. Acta (Amst.) 1960, $39,122$.

4. Milhaud, G., J.-P. Aubert, and J. Bourichon. Etude du métabolisme du calcium chez l'homme à l'aide de calcium 45. I. L'absorption du calcium au cours de la digestion. Path. et Biol. 1961, 9, 1761.

5. Bronner, F., and R. S. Harris. Absorption and metabolism of calcium in human beings, studied with calcium $^{45}$. Ann. N. Y. Acad. Sci. 1956, 64, 314.

6. Nordin, B. E. C. Investigation of bone metabolism with ${ }^{47} \mathrm{Ca}-\mathrm{a}$ preliminary report. Proc. roy. Soc. Med. 1959, 52, 351.

7. Krane, S. M., G. L. Brownell, J. B. Stanbury, and $H$. Corrigan. The effect of thyroid disease on calcium metabolism in man. J. clin. Invest. 1956, $35,874$.

8. Bauer, G. C. H., A. Carlsson, and B. Lindquist. Bone salt metabolism in humans studied by means of radiocalcium. Acta med. scand. 1957, 158, 143.

9. Dow, E. C., and J. B. Stanbury. Strontium and calcium metabolism in metabolic bone diseases. $\mathrm{J}$. clin. Invest. 1960, 39, 885.

10. Visek, W. J., R. A. Monroe, E. W. Swanson, and C. L. Comar. Determination of endogenous fecal calcium in cattle by a simple isotope dilution method. J. Nutr. 1953, 50, 23.

11. Comar, C. L., R. A. Monroe, W. J. Visek, and S. L. Hansard. Comparison of two isotope methods for determination of endogenous fecal calcium. J. Nutr. 1953, 50, 459.

12. Bauer, G. C. H., A. Carlsson, and B. Lindquist. Evaluation of accretion, resorption and exchange reactions in the skeleton. Kungliga Fysiografiska Sällskapet Lund, Förhandlingar 1955, 25, 1.

13. Bauer, G. C. H., A. Carlsson, and B. Lindquist. A comparative study on the metabolism of ${ }^{140} \mathrm{Ba}$ and ${ }^{45} \mathrm{Ca}$ in rats. Biochem. J. 1956, 63, 535. 
14. Bronner, F., R. S. Harris, C. J. Maletskos, and C. E. Benda. Studies in calcium metabolism. The fate of intravenously injected radiocalcium in human beings. J. clin. Invest. 1956, 35, 78.

15. Blau, M., H. Spencer, J. Swernov, and D. Laszlo. Utilization and intestinal excretion of calcium in man. Science 1954, 120, 1029.

16. Rich, C. The calcium metabolism of a patient with renal insufficiency before and after partial parathyroidectomy. Metabolism 1957, 6, 574.

17. Bauer, G. C. H., and R. D. Ray. Kinetics of strontium metabolism in man. J. Bone Jt Surg. 1958, 40A, 171.

18. Bronner, F., C. E. Benda, R. S. Harris, and J. Kreplick. Calcium metabolism in a case of gargoylism, studied with the aid of radiocalcium. J. clin. Invest. 1958, 37, 139.

19. Milhaud, G., and J.-P. Aubert. Etude des voies générales du métabolisme calcique chez l'homme in Radioisotopes in Scientific Research, R. C. Extermann, Ed. London, Pergamon, 1958, vol. 4, p. 120.

20. Bronner, F. Disposition of intraperitoneally injected calcium- 45 in suckling rats. J. gen. Physiol. 1958, 41, 767.

21. Solomon, A. K. Compartmental methods of kinetic analysis in Mineral Metabolism-An Advanced Treatise, C. L. Comar and F. Bronner, Eds. New York, Academic Press, 1960, vol. 1, part A, p. 119.

22. Bauer, G. C. H., A. Carlsson, and B. Lindquist. Metabolism and homeostatic function of bone in Mineral Metabolism-An Advanced Treatise, C. L. Comar and F. Bronner, Eds. New York, Academic Press, 1961, vol. 1, part B, p. 609.

23. Milhaud, G., W. Remagen, A. Gomes de Matos, and J.-P. Aubert. Etude du métabolisme du calcium chez le rat à l'aide de calcium 4 s. I. Le rachitisme expérimental. Rev. franç. Etud. clin. biol. 1960, $5,254$.

24. Aubert, J.-P., M. S. Moukhtar, and G. Milhaud. Etude du métabolisme du calcium chez le rat à l'aide de calcium 4 . III. Les relations entre les différents processus chez le rat normal. Rev. franç. Etud. clin. biol. 1961, 6, 1034.
25. Geissberger, W. Die Calciumresorption und Retention beim Menschen nach intravenöser, oraler und rectaler Calciumverabreichung mit Bilanzen unter Anwendung von radioaktivem Calcium. Z. ges. exp. Med. 1952, 119, 111.

26. Bronner, F. Experimental studies of calcium absorption in man in Modern Problems of Nutrition, J. C. Somogyi, Ed. Basel, S. Karger, 1962, vol. 3, p. 22.

27. Bronner, F., R. S. .Harris, C. J. Maletskos, and C. E. Benda. Studies in calcium metabolism. Effect of food phytates on calcium-45 uptake in children on low-calcium breakfasts. J. Nutr. 1954, 54, 523.

28. Bronner, F., J. R. Moor, R. S. Harris, and C. E. Benda. Calcium metabolism. V. Differential absorbability of calcium salts by children. Fed. Proc. 1955, 14, 428.

29. Marshall, J. H., R. E. Rowland, and J. Jowsey. Microscopic metabolism of calcium in bone. V. The paradox of diffuse activity and long-term exchange. Radiat. Res. 1959, 10, 258.

30. Neuman, W. F., and M. W. Neuman. The Chemical Dynamics of Bone Mineral. Chicago, University of Chicago Press, 1958,

31. Rich, C. The distribution of calcium given to human subjects by sustained intravenous infusion. J. clin. Endocr. 1960, 20, 147.

32. Rich, C., J. Ensinck, and H. Fellows. The use of continuous infusions of calcium- 45 and strontium- 85 to study skeletal function. J. clin. Endocr. 1961, 21, 611.

33. Bronner, F., P. D. Saville, and J. A. Nicholas. The Ca excretion index-a new parameter for metabolic studies utilizing $\mathrm{Ca}^{45}$. Fed. Proc. 1961, 20, 292.

34. Bronner, F., P. D. Saville, J. A. Nicholas, J. R. Cobb, P. D. Wilson, Jr., and P. D. Wilson. Calcium metabolism in man. Quantitation of calcium absorption and the excretion index in Radioisotopes and Bone, P. Lacroix and A. M. Budy, Eds. Oxford, Blackwell Scientific Publications, 1962, p. 17.

35. Bhandarkar, S. D., M. M. Bluhm, J. MacGregor, and B. E. C. Nordin. An isotope test of calcium absorption. Brit. med. J. 1961, 2, 1539. 